\title{
Searches for direct production of third generation squarks at CMS
}

\author{
Caroline Collard* \\ On behalf of the CMS Collaboration, \\ Université de Strasbourg, CNRS, IPHC UMR 7178, F-67000 Strasbourg, France \\ E-mail: caroline.collardecern.ch
}

\begin{abstract}
Searches for direct pair production of the third generation squarks (the supersymmetric partners of the top and bottom quarks) are presented, based on proton-proton collision data recorded by the CMS experiment during the LHC Run2 operations (2016-2018). These searches are performed in final states with one single lepton (electron or muon), a pair of tau leptons, or zero lepton. In absence of statistically significant deviations from the standard model background, the results are interpreted in the context of several decay modes of top squarks and for one decay mode of the bottom squarks.
\end{abstract}

European Physical Society Conference on High Energy Physics - EPS-HEP2019 -

10-17 July, 2019

Ghent, Belgium

${ }^{*}$ Speaker. 


\section{Introduction}

This report presents three different searches for direct pair production of the third generation squarks (the supersymmetric partners of the top and bottom quarks, namely the top squarks $\widetilde{t}$ and bottom squarks $\widetilde{b}$ ). The motivations for these searches are based on two observations. The first one is related to the Higgs boson discovery at a mass of $125 \mathrm{GeV}$ by the ATLAS and CMS Collaborations [1,2] and the fact that, in the Standard Model (SM), theoretical computations of the mass of the Higgs boson suffer from divergences of quantum loop corrections. Natural Supersymmetry scenarios can resolve this issue thanks to contributions involving supersymmetric particles, and in that case, the top and bottom squarks are expected to be light. The second important observation is the presence of dark matter in the universe. Working under R-parity conservation (RPC) allows the lightest supersymmetric particle (LSP) to be stable and it therefore could provide a candidate for dark matter. In the following, is only considered the case of the neutralino $\widetilde{\chi}_{1}^{0}$ to be the LSP. Another consequence of RPC is that supersymmetric particles are pair produced.

The three searches presented in this report are based on a different lepton content in the final state. The analyses requesting the presence of either one single lepton [3] or a pair of tau leptons [4] are optimized to target the direct production of $\widetilde{\tilde{t}}$, addressing different possible decay chains for the top squark up to the LSP. The last analysis [5] is an inclusive search with zero lepton in the final state, leading to an interpretation for both the productions of $\widetilde{\tilde{t} t}$ and $\tilde{b} \bar{b}$. All these searches are performed using the data collected by the CMS detector [6] during the LHC Run2 operations (2016-2018).

\section{Search for top squarks with one lepton in the final state}

The results of the search for top squarks with one lepton in the final state [3] have just been released in time for the EPS-HEP2019 conference. This analysis is based on the full Run2 data set, corresponding to an integrated luminosity of $137 \mathrm{fb}^{-1}$, and targets three different scenarios for the decay of the top squarks as represented on Figure 1.
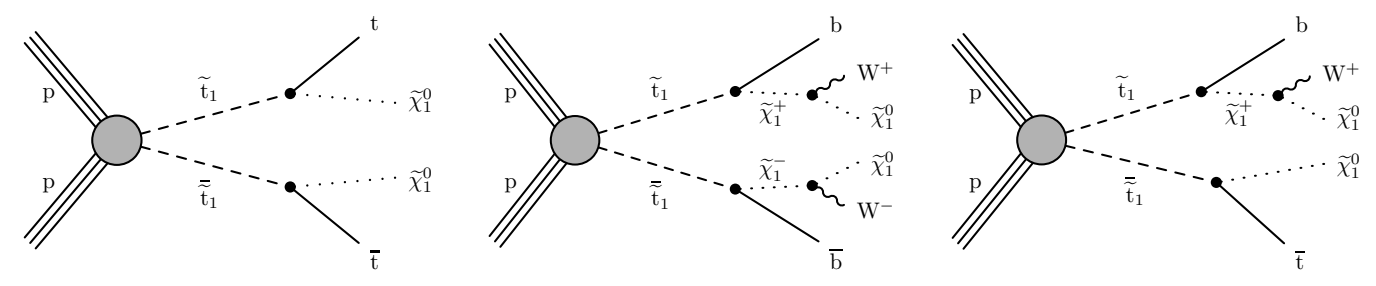

Figure 1: Diagrams of production and decay of the top squarks considered in Ref. [3].

The preselection requests exactly one lepton $(\ell=e$ or $\mu)$, at least two jets among which at least one should pass some b-tagging criteria, a large missing transverse energy $\left(E_{\mathrm{T}}^{\text {miss }}\right)$, and $M_{\mathrm{T}}=$ $\sqrt{2 p_{\mathrm{T}}(\ell) E_{\mathrm{T}}^{\text {miss }}\left(1-\Delta \Phi\left(\ell, E_{\mathrm{T}}^{\text {miss }}\right)\right)}>150 \mathrm{GeV}$ where $p_{\mathrm{T}}(\ell)$ is the transverse momentum $\left(p_{\mathrm{T}}\right)$ of the lepton and $\Delta \Phi\left(\ell, E_{\mathrm{T}}^{\text {miss }}\right)$ the angle in the transverse plane between the lepton and the $E_{\mathrm{T}}^{\text {miss }}$ direction. While following the same strategy as in Ref. [7] which was based on $35.9 \mathrm{fb}^{-1}$ of data, this new 
analysis also introduces new techniques in order to address specific areas. For example, the $\mathrm{W}$ corridor phase space, defined by a difference in mass $\Delta m=m(\widetilde{t})-m\left(\widetilde{\chi}_{1}^{0}\right)$ between the top squark and the LSP, close to the mass of the $\mathrm{W}$ boson, is challenging because of the softness (lower $p_{\mathrm{T}}$ ) of the decay products. In order to enhance the sensitivity of the analysis here, a soft b-tagging algorithm [8] replaces the standard one (deepCSV [9]) in the selection, and the low jet multiplicity case is considered. In the high $m(\widetilde{t})$ regime, hadronically decaying top quarks are identified using two separate taggers based on deep neural networks: one addressing the moderate $p_{\mathrm{T}}$ boost with three separated jets ("resolved" top tagger) and the other one for large $p_{\mathrm{T}}$ boost where the decay products are merged into a wide jet ("merged" top tagger [10]).

First, 39 Search Regions (SR) are defined in bins of jet multiplicity $\left(N_{J}\right)$, the topness variable $\left(t_{\mathrm{mod}}\right)$ telling how well the event agrees with the hypothesis of a $\bar{t} \bar{t}$ pair decaying fully leptonically, the mass of the lepton and closest b-tagged jet system $\left(M_{\ell b}\right), E_{\mathrm{T}}^{\text {miss }}$, and for the top categories (merged, resolved, un-tagged top) or inclusively. In addition, a specific selection is applied in the top corridor (with $\Delta m$ close to the mass of the top quark), requesting $N_{J} \geq 5$, the leading jet not being b-tagged and a low $p_{\mathrm{T}}$ lepton with a $p_{\mathrm{T}}(\ell)$ cut depending on $\Delta \Phi\left(\ell, E_{\mathrm{T}}^{\mathrm{miss}}\right)$. For this corridor, 5 $\mathrm{SR}$ are defined in bins of $E_{\mathrm{T}}^{\text {miss }}$. The selection for the $\mathrm{W}$ corridor is the same as for the top corridor but requesting $N_{J} \geq 3$ and using the soft b-tagging algorithm in place of the standard algorithm.

The observed yields in each SR are displayed on Figure 2. The contributions from the different backgrounds are also represented. The dominant one ("Lost lepton") is coming from t $\bar{t}$ and tW processes with 2 leptons in the decay chains but for which one lepton is lost. Then the background with one single lepton is mainly coming from $\mathrm{W}+\mathrm{jets}$ (" $1 \ell$ not from top") while the contribution from processes including top (" $1 \ell$ from top") is much smaller. The tt̄Z process (and to a less extend $\mathrm{WZ}, \ldots)$, with the $\mathrm{Z}$ boson decaying invisibly $(\mathrm{Z} \rightarrow v v)$, also plays a role in the background list.

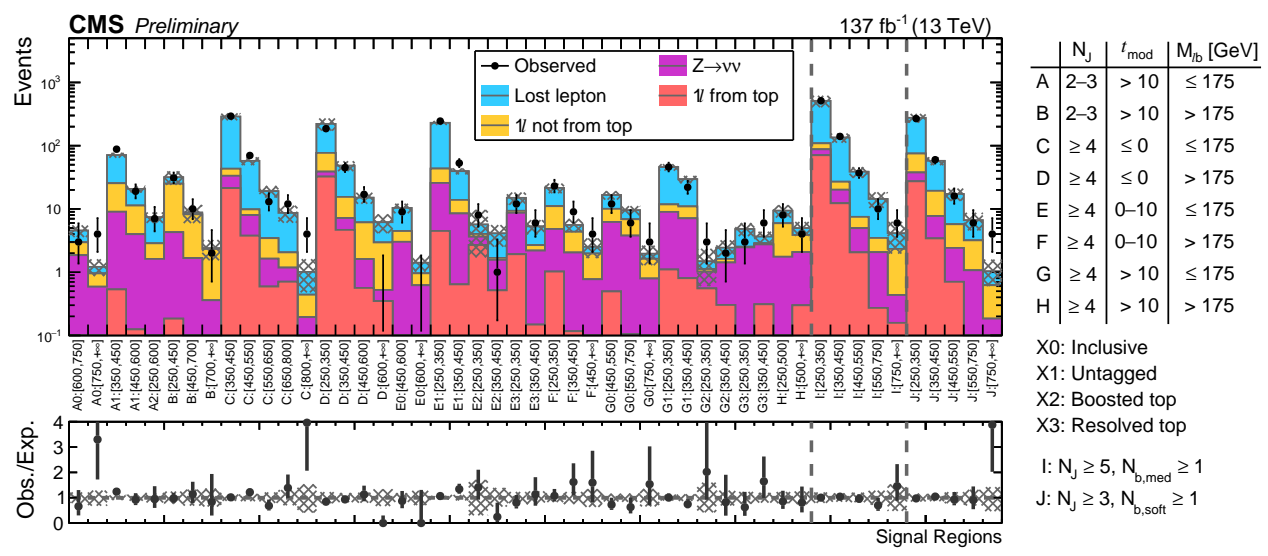

Figure 2: Observed and expected yields in the different SR of the $\tilde{t}$ search in the 1-lepton final state [3]. The lower panel shows the ratio of the observed yield to the expected one. The shaded bands correspond to the statistical and systematic uncertainties added in quadrature.

The observed yields being consistent with the expectations from the SM background, the results have been interpreted for different $\widetilde{t}$ decay scenarios. The exclusion limits are presented on Figure 3. In the case where top squarks always decay into a top quark and a neutralino, masses of $\tilde{t}$ are excluded up to $1200 \mathrm{GeV}$ for a massless $\widetilde{\chi}_{1}^{0}$. A large gain is also obtained with respect to the 
previous search [7] at low $\Delta m$ by considering the W-corridor SR. The other interpretations use only the 39 first SR and show a gain of $\sim 100 \mathrm{GeV}$ in $m(\widetilde{t})$ at low $m\left(\widetilde{\chi}_{1}^{0}\right)$ with respect to [7].
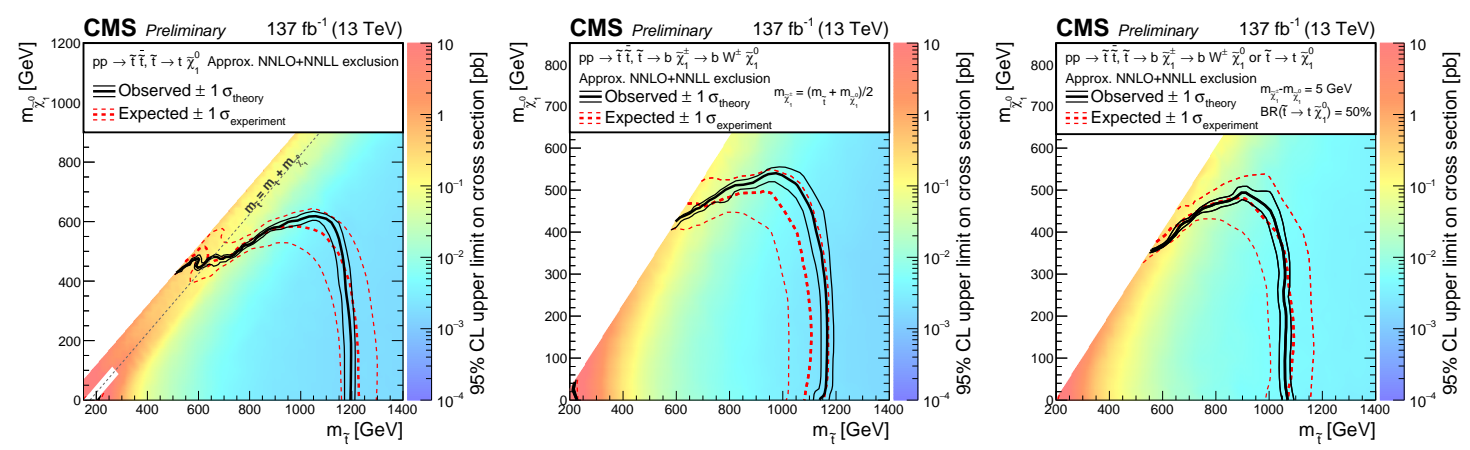

Figure 3: Exclusion limits at 95\% CL for direct $\widetilde{t}$ production in the three decay scenarios considered on Figure 1. The thick black curve corresponds to the observed limit, while the thick and dashed red one to the expected limit. The thin curves present the impact of one standard deviation uncertainties, in black from theory and in red from experimental uncertainties [3].

\section{Search for top squarks in a final state with two tau leptons}

The results of the search for top squarks in a final state with two tau leptons [4] was released at the time of the LHCP2019 conference. Based on $77.2 \mathrm{fb}^{-1}$ of data, this analysis considers for the first time event signatures with two hadronically decaying tau leptons and at least one b-tagged jet in a $\tilde{t}$ search. One of the four diagrams considered in the analysis is presented on Figure 4 (left), illustrating the $\widetilde{t}$ decay into the lightest chargino $\widetilde{\chi}_{1}^{ \pm}$and a b quark, then followed by the $\widetilde{\chi}_{1}^{ \pm}$decay into a tau slepton $\widetilde{\tau}$ and a neutrino or a sneutrino $\widetilde{v}_{\tau}$ and a tau lepton, with an equal probability. The mass assumptions used in these scenarios are the following: $m\left(\widetilde{\chi}_{1}^{ \pm}\right)-m\left(\widetilde{\chi}_{1}^{0}\right)=0.5\left(m(\widetilde{t})-m\left(\widetilde{\chi}_{1}^{0}\right)\right)$, $m(\widetilde{\tau})-m\left(\widetilde{\chi}_{1}^{0}\right)=x\left(m\left(\widetilde{\chi}_{1}^{ \pm}\right)-m\left(\widetilde{\chi}_{1}^{0}\right)\right), m(\widetilde{\tau})=m\left({\widetilde{v_{\tau}}}\right)$, with $x=0.25,0.5$ or 0.75 . In total 15 SR are defined, categorised in bins of $H_{\mathrm{T}}=\Sigma p_{\mathrm{T}}$ (jets with $\left.|\eta|<2.4\right)+\Sigma p_{\mathrm{T}}(\tau)$, the missing transverse momentum $p_{\mathrm{T}}^{\text {miss }}$ and the stransverse mass $\left(M_{\mathrm{T} 2}\right)$ which is a generalisation of the $M_{\mathrm{T}}$ variable when there are multiple sources of $p_{\mathrm{T}}^{\text {miss }}$. No significative deviation is observed with respect to the SM prediction, with backgrounds originating from misidentified jets as $\tau$ lepton, or from $t \bar{t}$ decaying into genuine $\tau$ leptons as well as from $\mathrm{Z}+\mathrm{jets}$ and other rare SM processes. The results are then interpreted for different values of $x$. Only the $x=0.5$ case is shown on Figure 4 (right), the other exclusion limits being similar.

\section{Inclusive search in multijet events with large missing transverse momentum}

The third analysis [5] presented in this report has also delivered new results for the EPS$H E P 2019$ conference. Based on the full Run2 statistics (corresponding to an integrated luminosity of $137 \mathrm{fb}^{-1}$ ), the search is performed in the 0-lepton channel, requesting $N_{J} \geq 2, H_{\mathrm{T}}=\Sigma p_{\mathrm{T}}$ (jets with $|\eta|<2.4)>300 \mathrm{GeV}$ and $H_{\mathrm{T}}^{\text {miss }}=\mid \Sigma \vec{p}_{\mathrm{T}}$ (jets with $\left.|\eta|<5\right) \mid>300 \mathrm{GeV}$. Among all the different simplified model spectra (SMS) targetted by this analysis, the $\widetilde{t}$ and $\widetilde{b}$ pair productions are 

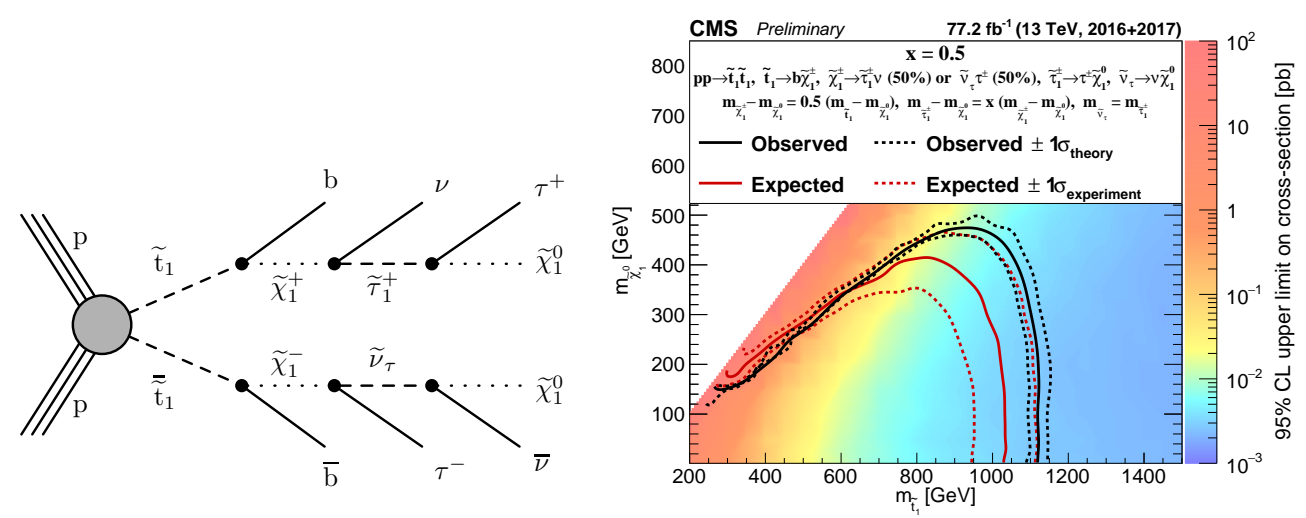

Figure 4: (left) One of the four decay chains considered in this analysis. (right) Exclusion limits at 95\% CL for direct $\widetilde{t}$ pair production leading to two $\tau$ leptons in the final state, with $x=0.5$ [4].

considered, followed by the $\widetilde{t} \rightarrow t+\widetilde{\chi}_{1}^{0}$ and $\widetilde{b} \rightarrow b+\widetilde{\chi}_{1}^{0}$ decays, respectively. To cover widely all these SMS, $174 \mathrm{SR}$ are defined in bins of $N_{J}$, the number of b-tagged jets $\left(N_{b}\right), H_{\mathrm{T}}$ and $H_{\mathrm{T}}^{\text {miss }}$. The observed and expected yields in each of these SR are displayed on Figure 5 (left). The backgrounds are composed of events from $t \bar{t}$ or $\mathrm{W}+$ jets with one lost lepton, $\mathrm{Z}+\mathrm{jets}$ with $\mathrm{Z} \rightarrow v v$, and QCD multijets. The $95 \%$ CL exclusion limits for the $\widetilde{b}$ pair production is displayed on Figure 5 (right). Masses of $\widetilde{b}$ of $1220 \mathrm{GeV}$ are excluded for almost massless $\widetilde{\chi}_{1}^{0}$, with a gain greater than $200 \mathrm{GeV}$ with respect to the previous analysis [11] based on $35.9 \mathrm{fb}^{-1}$. Similarly, $\widetilde{t}$ with masses up to $1190 \mathrm{GeV}$ are excluded for massless $\widetilde{\chi}_{1}^{0}$.
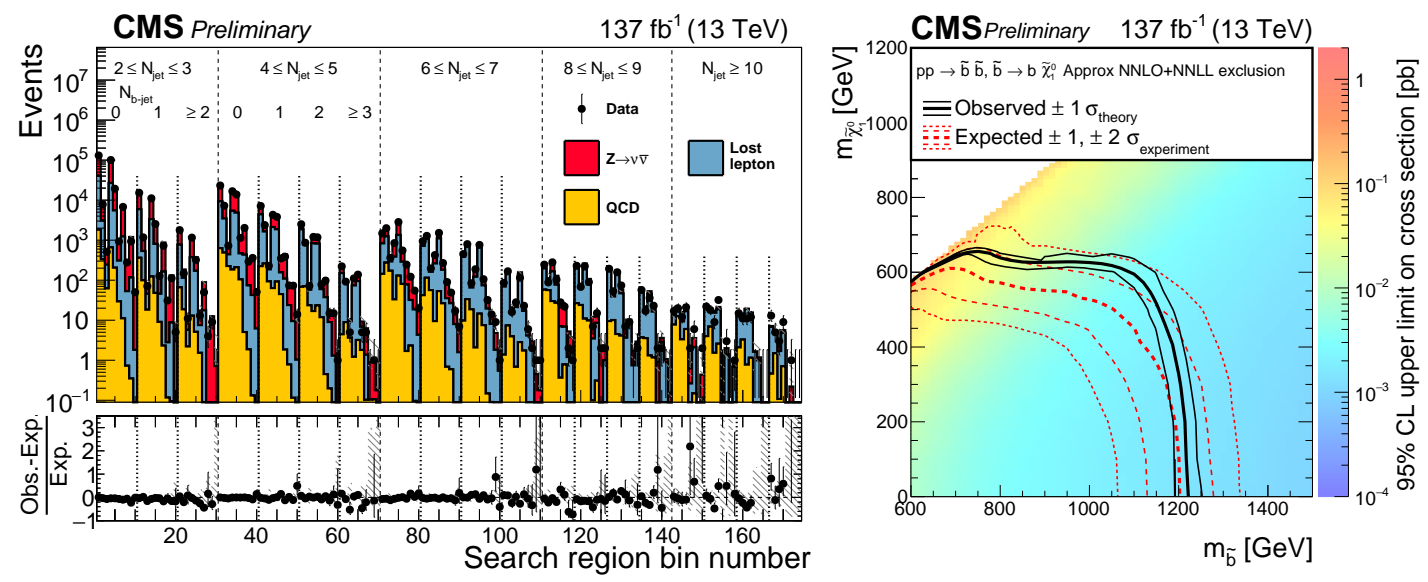

Figure 5: (left) Observed and expected yields in the different SR of the 0-lepton analysis. (right) Exclusion limits at $95 \%$ CL for direct $\widetilde{b}$ pair production [5].

\section{A last word}

No sign of physics beyond the Standard Model has been discovered yet, but plenty of searches are ongoing, looking for different final states, taking advantage of the full Run2 luminosity, devel- 
oping new ideas or new techniques, ... For sure, more results are expect to come on the subject, so stay tuned!

\section{Acknowledgements}

I would like to warmly thank the organizers for this very pleasant and interesting conference.

\section{References}

[1] G. Aad et al. [ATLAS Collaboration], "Observation of a new particle in the search for the Standard Model Higgs boson with the ATLAS detector at the LHC", Phys. Lett. B 716 (2012) 1, doi:10.1016/j.physletb.2012.08.020 [arXiv:1207.7214 [hep-ex]].

[2] S. Chatrchyan et al. [CMS Collaboration], "Observation of a new boson at a mass of $125 \mathrm{GeV}$ with the CMS experiment at the LHC", Phys. Lett. B 716 (2012) 30, doi:10.1016/j.physletb.2012.08.021 [arXiv:1207.7235 [hep-ex]].

[3] CMS Collaboration, "Search for direct top squark pair production in events with one lepton, jets and missing transverse energy at $13 \mathrm{TeV}$ ", CMS SUS-19-009, https://cds.cern.ch/record/2682157.

[4] CMS Collaboration, "Search for top squark pair production in a di-tau final state in proton-proton collisions at $\sqrt{s}=13 \mathrm{TeV}$ ", CMS SUS-19-003, https://cds.cern.ch/record/2676094.

[5] CMS Collaboration, "Search for supersymmetry in proton-proton collisions at $13 \mathrm{TeV}$ in final states with jets and missing transverse momentum", CMS SUS-19-006, https://cds.cern.ch/record/2682103.

[6] S. Chatrchyan et al. [CMS Collaboration], "The CMS Experiment at the CERN LHC", JINST 3 (2008) S08004, doi:10.1088/1748-0221/3/08/S08004.

[7] A. M. Sirunyan et al. [CMS Collaboration], "Search for top squark pair production in pp collisions at $\sqrt{s}=13 \mathrm{TeV}$ using single lepton events", JHEP 1710, 019 (2017), doi:10.1007/JHEP10(2017)019, [arXiv: 1706.04402 [hep-ex]].

[8] A. M. Sirunyan et al. [CMS Collaboration], "Search for direct production of supersymmetric partners of the top quark in the all-jets final state in proton-proton collisions at $\sqrt{s}=13 \mathrm{TeV}$ ", JHEP 1710, 005 (2017), doi:10.1007/JHEP10(2017)005, [arXiv:1707.03316 [hep-ex]].

[9] A. M. Sirunyan et al. [CMS Collaboration], "Identification of heavy-flavour jets with the CMS detector in pp collisions at 13 TeV", JINST 13 (2018) P05011, doi:10.1088/1748-0221/13/05/P05011, [arXiv:1712.07158 [hep-ex]].

[10] CMS Collaboration, "Machine learning-based identification of highly Lorentz-boosted hadronically decaying particles at the CMS experiment", CMS-PAS-JME-18-002,

https://cds.cern.ch/record/2683870.

[11] A. M. Sirunyan et al. [CMS Collaboration], "Search for supersymmetry in multijet events with missing transverse momentum in proton-proton collisions at 13 TeV", Phys. Rev. D 96 (2017) 032003, doi:10.1103/PhysRevD.96.032003, [arXiv:1704.07781[hep-ex]]. 